\title{
Geared Linkage driven by Linear Actuator used for PV Platform Azimuth Orientation
}

\author{
N.C. Creanga ${ }^{1}$, I. Visa $^{1}$, D.V. Diaconescu ${ }^{1}$, I.S. Hermenean $^{1}$ and B.R. Butuc $^{1}$ \\ ${ }^{1}$ Department Renewable Energy Systems and Recycling \\ Transilvania University of Brasov \\ B-ul Eroilor, 29, 500036 Brasov (Romania)
}

Phone/Fax number:+40-(268) 41.20.88, +40-(268) 41.05.25, e-mail: \{nora.creanga, visaion, dvdiaconescu, ioana.hermenean, bianca.butuc\}@unitbv.ro

\begin{abstract}
To gain more solar energy the photovoltaic converter must be tracked relatively to the sun movement. The majority of the tracking systems use complicated and costly rotating mechanisms to build large angular strokes. This paper presents a new type of geared linkage composed of a triangular linkage fitted with a planetary gear pair able to reach high angular strokes. This linkage is used as basic mechanism for the azimuth tracking of a photovoltaic platform. The kinematic synthesis of the linkage shows that the azimuth angular stroke increases with the planetary gear internal ratio $\left|i_{0}\right|$. The linkage is further implemented for an azimuth mono-axial tracked PV module located in a mountain location (case study - Brasov, Romania). Through numerical simulations, the amount of direct solar radiation on the $P V$ module and the tracking efficiency are evaluated.
\end{abstract}

\section{Key words}

Photovoltaic module, azimuth tracking system, geared linkage, large angular strokes, tracking efficiency.

\section{Introduction}

Due to the alarming increase in $\mathrm{CO} 2$ emissions, the major cause of global warming, the use of renewable energy sources has become a must. Photovoltaic systems represent a successful path, providing green energy by using the (direct) solar radiation, [1]. To improve their efficiency, tracking systems are imposed. These have the role of following the sun path throughout the day, thus increasing the amount of direct radiation that falls normal on the PV module surface [2].

The tracking systems designed nowadays mainly use rotating actuators to accomplish large angular strokes [3]. The disadvantage of such systems is their high complexity and their cost. An alternative to this system is the linear actuator solution, having the drawback of small transmission angles, thus small angular strokes.

One existing solution is a two-contour linkage [4] in which a four bars mechanism of $4 R$ type $(R=$ revolute joint) performs high angular strokes (approximately $180^{\circ}$ ) due to the amplification delivered by a linear actuator.
Our research focuses on the development of a new azimuth tracking mechanism (Figure 1) which performs larger angular strokes on a better transmission angle. The main objective is to increase the amount of direct solar radiation perpendicularly falling on the photovoltaic surface, by using this type of mechanism.

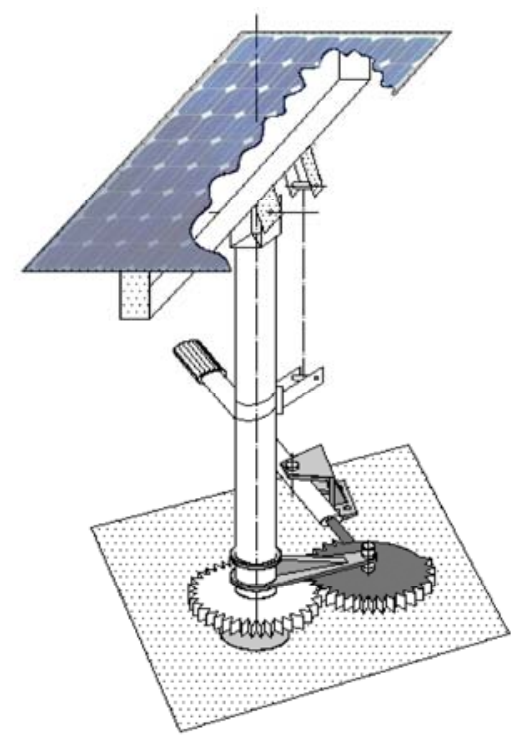

a)

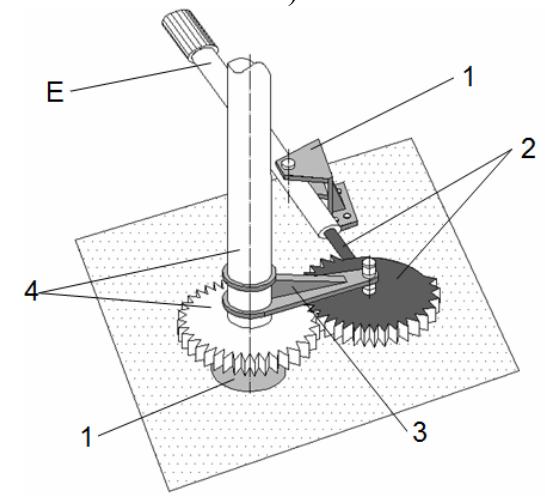

b)

Fig. 1. a) PV system with tracking geared linkage b) geared linkage components. 
For modeling, the mechanism is further included in an azimuth mono-axial tracking system implemented in a mountain region, for the case study this location is Brasov, Romania, considering all the local geographic and meteorological parameters.

\section{Kinematical Synthesis}

The new type of mono-axial tracking system is build up of a geared mechanism (Figure 1 and 2), which is obtained by adding a differential planetary gear pair (Figure 3) to a triangle type linkage (Figure 4).

The components of this geared linkage are (Figure 1.b and 2): a base $(A B=b)$, a rocker $(B C=r)$ and gears 2 and 4 . Gear 4 is connected and fixed to an azimuth pole and gear 2 is connected and fixed to a linear actuator element (E).

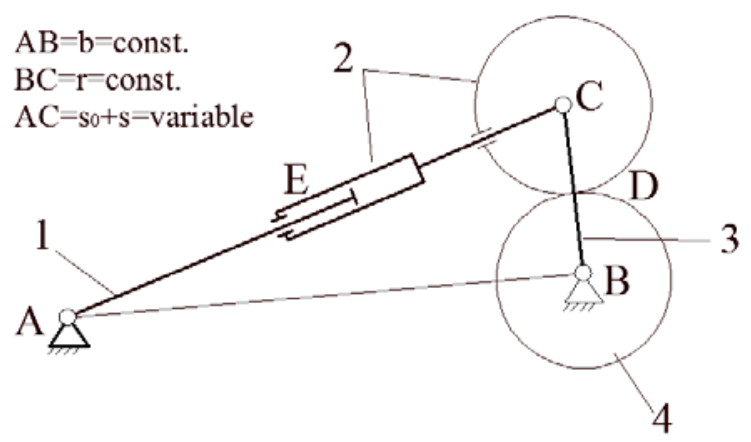

Fig. 2. Geared linkage structure.

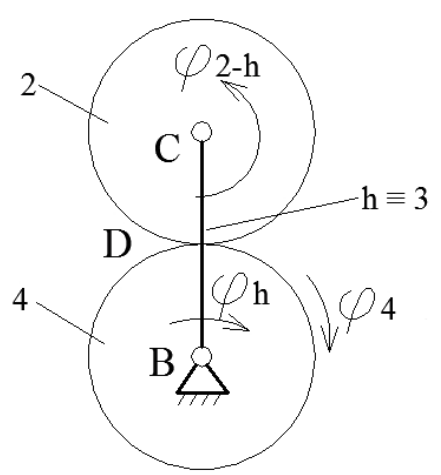

a)

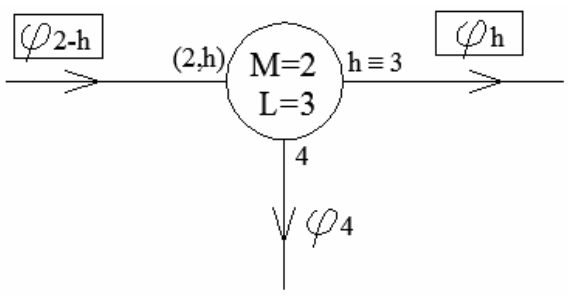

b)

Fig. 3. Differential planetary gear pair (a) and its black box scheme (b).

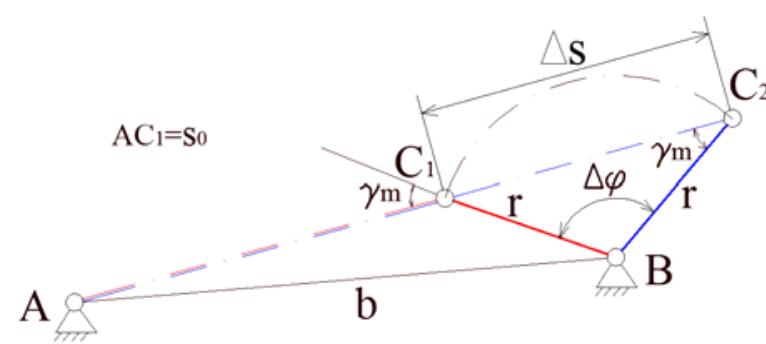

Fig. 4. Initial and final position of the linkage for a minimum admitted value of the transmission angle $\left(\gamma_{\mathrm{m}}\right)$.

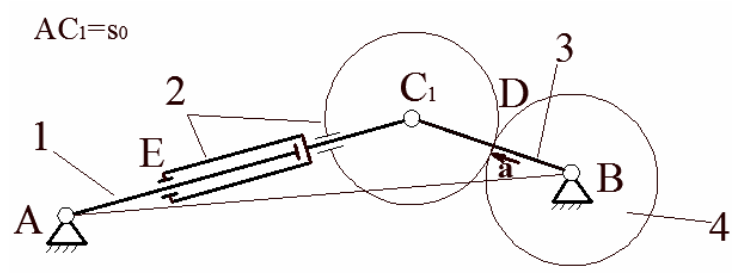

a)

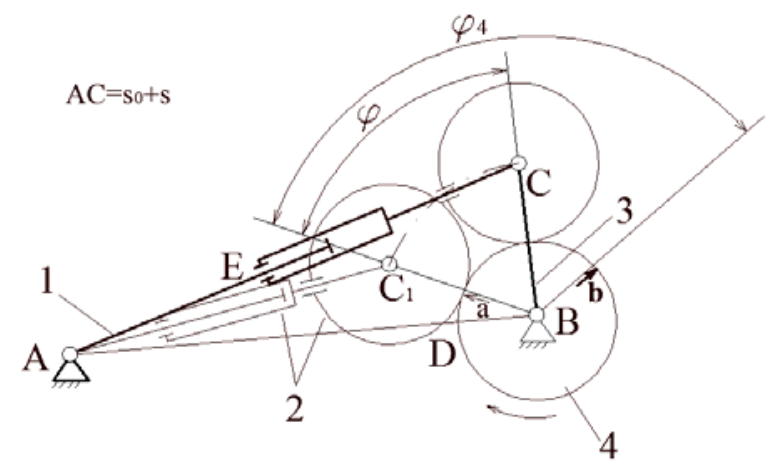

b)

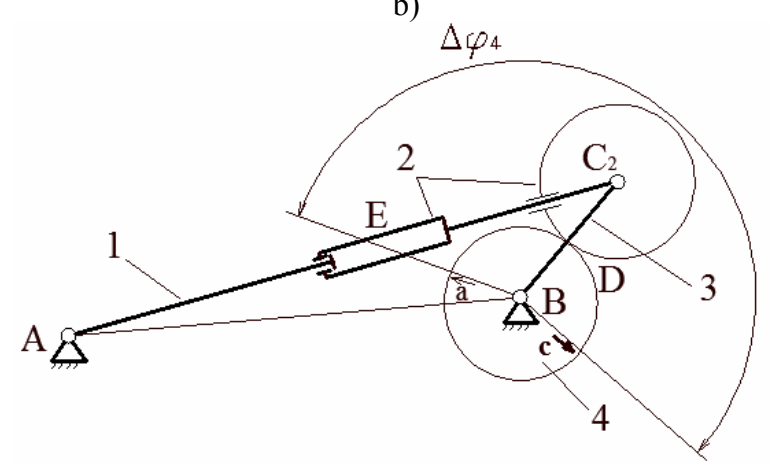

c)

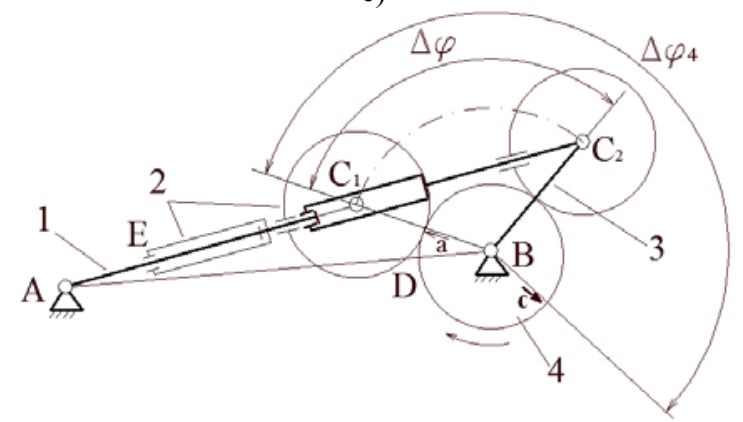

d)

Fig. 5. a) Initial position (a) of the geared linkage; b) the initial (a) and a current position (b) of the geared linkage; c) final position (c) of the geared linkage; d) the initial (a) and final position (c) of the geared linkage. 
Applying the Sinus Theorem in Figure 4, the following equations are found:

$$
\begin{gathered}
\frac{b}{\sin \gamma_{m}}=\frac{r}{\sin \left(C_{2} A B\right)}=\frac{A C_{2}}{\sin \left(A B C_{2}\right)}, \\
C_{2} A B=\arcsin \left((r / b) \cdot \sin \gamma_{m}\right), \\
\frac{b}{\sin \gamma_{m}}=\frac{r}{\sin \left(C_{1} A B\right)}=\frac{A C_{1}}{\sin \left(A B C_{1}\right)}, \\
\angle \mathrm{C}_{1} \mathrm{AB}=\arcsin \left((\mathrm{r} / \mathrm{b}) \cdot \sin \gamma_{\mathrm{m}}\right)=\angle \mathrm{C}_{2} \mathrm{AB}
\end{gathered}
$$

Based on the above equations, the rocker's angular stroke $\Delta \varphi=\angle \mathrm{C}_{1} \mathrm{BC}_{2}$ (Figure 5.d) and the stroke $\Delta \mathrm{s}=\mathrm{C}_{1} \mathrm{C}_{2}$ (Figure 4) of the linear actuator are found, considering a minimum value for the transmission angle $\left(\gamma_{\mathrm{m}}\right)$ :

$$
\begin{aligned}
& \Delta \varphi=180^{\circ}-2 \cdot \gamma_{m}, \\
& \Delta s=2 \cdot r \cdot \cos \gamma_{m},
\end{aligned}
$$

In which the difference $\Delta$ means a displacement stroke (i.e. the difference between the end and start values)

The internal speed ratio of the differential planetary mechanism is defined by the equation (Figure 3.a):

$$
\begin{gathered}
i_{0}=i_{4-2}^{h}=\frac{\varphi_{4-h}}{\varphi_{2-h}}=\frac{\Delta \varphi_{4-h}}{\Delta \varphi_{2-h}}=-\frac{z_{2}}{z_{4}}, \\
\Delta \varphi_{4-h}=\Delta \varphi_{4}-\Delta \varphi_{h}=\Delta \varphi_{4}-\Delta \varphi \\
\varphi_{4-h}=\varphi_{4}-\varphi_{h}=\varphi_{4}-\varphi .
\end{gathered}
$$

The first ratio from the equation (7) is valid only if the start values of the considered angles are null; this condition is achieved for the analytical models of the proposed gear linkage (Figures 3, 4 and 5).

Considering the equations (7) and ( $\left.7^{\prime}\right)$, Figure 4 and $3 a$ the gear linkage analytic model can be found:

$$
\begin{gathered}
\Delta \varphi_{4}=i_{0} \cdot \Delta \varphi_{2-3}+\Delta \varphi, \\
\Delta \varphi_{2-3}=\Delta \varphi_{2-h}=2 \cdot \gamma_{m}-180^{\circ}<0 \\
\Delta \varphi=C_{1} B C_{2}=180^{\circ}-2 \cdot \gamma_{m}>0, \\
\Delta \varphi_{4}=i_{0} \cdot\left(2 \gamma_{m}-180^{\circ}\right)+180^{\circ}-2 \gamma_{m},
\end{gathered}
$$

where: $\Delta \varphi_{4}$ represents the angular stroke of the azimuth gear (Figure 5c. and 5.d) and $\Delta \varphi_{2-3}$ is the angular stroke of the rocker 3 relative to gear 2 .

Figure 5 shows the initial position of the azimuth gear symbolized through (a), a current position of the azimuth gear symbolized through (b) and the final position of azimuth gear symbolized through (c).
The $\mathrm{b} / \mathrm{r}$ ratio of the geared linkage depends on the ratio $k_{a}=A C_{1} / r$ (Figures 4 and 7 ):

$$
(b / r)^{2}=1+k_{a}^{2}+2 \cdot k_{a} \cdot \cos \gamma_{m}
$$

The kinematical study of the geared linkage shows that, compared to the angular stroke of the triangle type linkage (Figure 4), larger angular strokes are obtained, considering the internal speed ratio $\mathrm{i}_{0}$ (Figure 5). As exemple, the following hypothetical in-practice demands are considered:

a) tracker's angular stroke (Figure 2 and 3): $\Delta \varphi_{4}=200^{\circ}$;

b) admitted minimum transmission angle: $\gamma_{\mathrm{m}} \geq 30^{\circ}$;

c) rocker's length (resulted from system's resistance to wind loads): $r=300 \mathrm{~mm}$ (Figure 4);

d) actuator's minimum length (Figure 4): $\mathrm{s}_{0}=\mathrm{AC}_{1}=$ $180 \mathrm{~mm} \quad\left(k_{a}=\mathrm{AC}_{1} / r=0.6\right)$;

e) actuator's stroke (Figure 4): $\Delta s=\mathrm{C}_{1} \mathrm{C}_{2}=500 \mathrm{~mm}$.

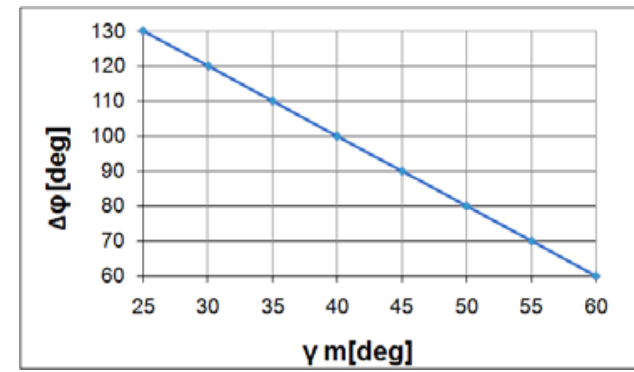

a)

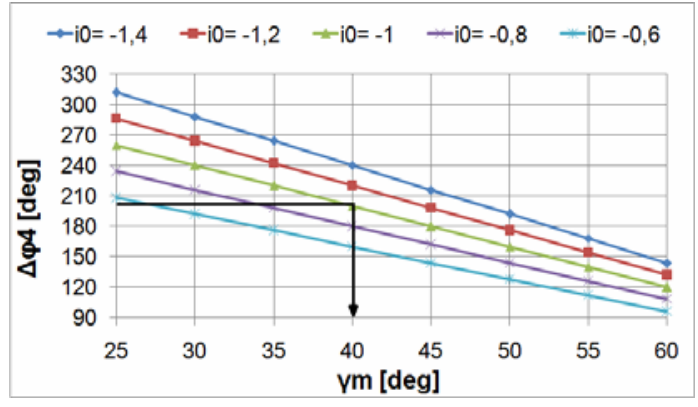

b)

Fig. 6. Angular stroke variations: a) of the triangle type linkage and $b$ ) of the geared linkage with different ratio $i_{0}$.

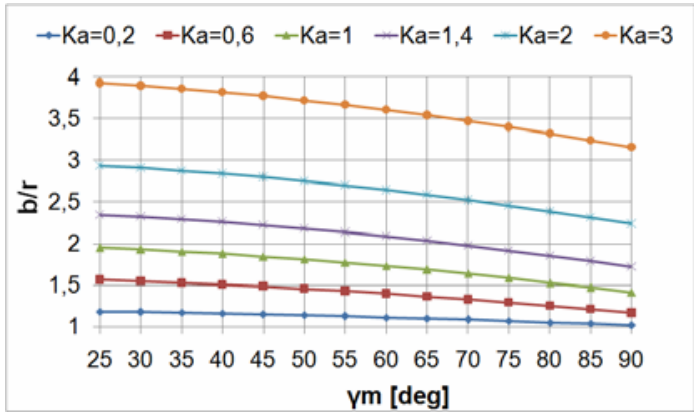

Fig. 7. Variations of the ratio $b / r$ for different values of $k_{a}$.

Based on this example, a synthesis algorithm in two steps is further developed. 
Step 1: Estimating the values of the sizes $i_{0}, \gamma_{m}$ and $\Delta \varphi$, vs. the imposed size $\Delta \varphi_{4}$ :

Based on Figure 6 and on the imposed value $\Delta \varphi_{4}$, the values for the $\mathrm{i}_{0}$ ratio (with discret variations of $-1.4,-1.2$, $-1,-0.8$ and -0.6 ), for the minimum transmission angle $\gamma_{\mathrm{m}}$ (with values from $25^{\circ}$ to $60^{\circ}$ ) and for the linkage stroke $\Delta \varphi$ can be estimated.

Table I. - Examples of synthesis solutions

\begin{tabular}{|c|c|c|c|c|c|}
\hline \multicolumn{6}{|c|}{ Input dates: } \\
\hline \multicolumn{6}{|c|}{$\begin{array}{l}\Delta \varphi_{4}=200^{\circ} ; \gamma_{\text {min.ad. }}=30^{\circ} \\
r=300 \mathrm{~mm} ; k_{a}=\mathrm{AC}_{1} / r=0.6 \\
s=\mathrm{AC}_{2}-\mathrm{AC}_{1}=500 \mathrm{~mm}\end{array}$} \\
\hline \multicolumn{6}{|c|}{ Results: } \\
\hline$i_{0}$ & $\gamma$ & $k_{a}$ & $b / r$ & $b$ & $S$ \\
\hline- & {$\left[{ }^{\circ}\right]$} & - & - & \multicolumn{2}{|c|}{$[\mathrm{mm}]$} \\
\hline \multirow{6}{*}{-1} & \multirow{6}{*}{40} & 0.2 & 1.16 & 348.10 & 459.62 \\
\hline & & 0.6 & 1.51 & 452.91 & 459.62 \\
\hline & & 1 & 1.87 & 563.81 & 459.62 \\
\hline & & 1.4 & 2.25 & 677.82 & 459.62 \\
\hline & & 2 & 2.83 & 851.92 & 459.62 \\
\hline & & 3 & 3.82 & 1146.15 & 459.62 \\
\hline
\end{tabular}

Step 2: Calculating the $r / b$ ratio

Using the data in Figure 7, the $r / b$ ratio (depending on the ratio $k_{a}$ ) can be evaluated, for each previous estimated pair of values sets for $i_{0}$ and $\gamma_{\mathrm{m}}$.

In Table 1, there are presented only 6 of the possible solutions, while the rest of the solutions can be estimated through interpolation.

According to Table 1, the most adequate stroke (considering the imposed value of $500 \mathrm{~mm}$ ) is $\mathrm{s}=459.62 \mathrm{~mm}$; therefore, the required geared linkage with $\mathrm{b}>\mathrm{r}$ (Figure 2) works on the following parameters: $\gamma_{\mathrm{m}}=40^{\circ} ; \mathrm{i}_{0}=-1 ; \mathrm{k}_{\mathrm{a}}=0.6 ; \mathrm{r}=300 \mathrm{~mm}$ and $\mathrm{b}=452.91 \mathrm{~mm}$.

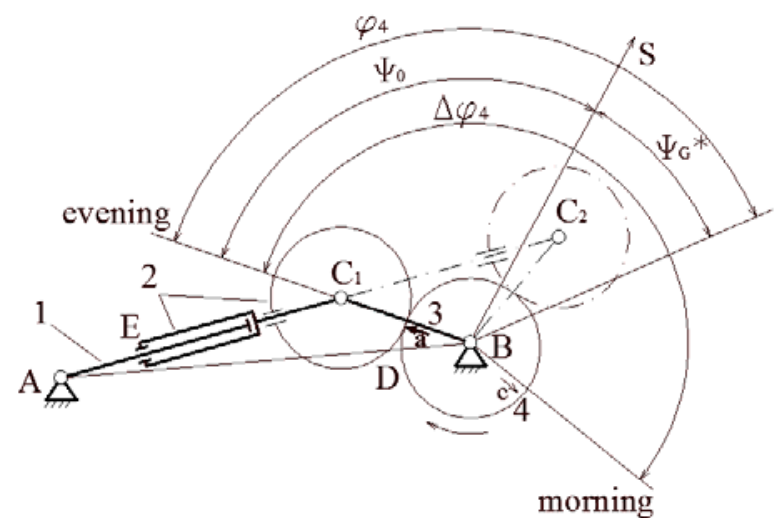

Fig. 8. Initial and final positions of the geared linkage considering the South orientation.

According to Figure 8, where $\mathrm{S}$ indicates the South direction and gear wheel 4 is a part of the PV pole, the correlation between the angular displacement of the geared linkage $\left(\varphi_{4}\right)$ and the azimuth angular displacement of the same device $\left(\psi_{\mathrm{G}}{ }^{*}\right)$ is given by the equation:

$$
\psi_{\mathrm{G}}^{*}=\varphi_{4}-\psi_{0}=\varphi_{4}-\left(\Delta \varphi_{4}\right) / 2
$$

where: $\psi_{\mathrm{G}}^{*}$ is the geared linkage azimuth current angle (i.e. the angle between South direction and the current position of the gear 4$) ; \varphi_{4}$ represents the previous current angle of the gear 4 and $\psi_{0}=\left(\Delta \varphi_{4}\right) / 2$ is the angle between the two starting positions (Figure 8): $(\mathrm{a})=\mathrm{BC}_{1}$ and South direction.

\section{Numerical Simulations and Results}

Based on the numerical data in Table 1 and equations 7 and 7 ', for the displacement transmission function $\varphi_{4}=$ $\varphi_{4}(\mathrm{~s})$ following equation is obtained:

$$
\varphi_{4}=\mathrm{i}_{0} \cdot \varphi_{2-3}+\varphi
$$

where: $\varphi$ and $\varphi_{2-3}$ values (depending on the actuator linear displacement s) can be found from Figure 5b, as:

$$
\begin{gathered}
\varphi_{2-3}=\angle \mathrm{ACB}-\angle \mathrm{AC}_{1} \mathrm{~B} \\
\varphi=\angle \mathrm{ABC}-\angle \mathrm{ABC}_{1}
\end{gathered}
$$

According to Figure 9 and using the equation (13), the azimuth displacement function $\psi_{\mathrm{G}}{ }^{*}=\psi_{\mathrm{G}} *(\mathrm{~s})$ of the PV platform is derived from curve $\varphi_{4}=\varphi_{4}(\mathrm{~s})$.

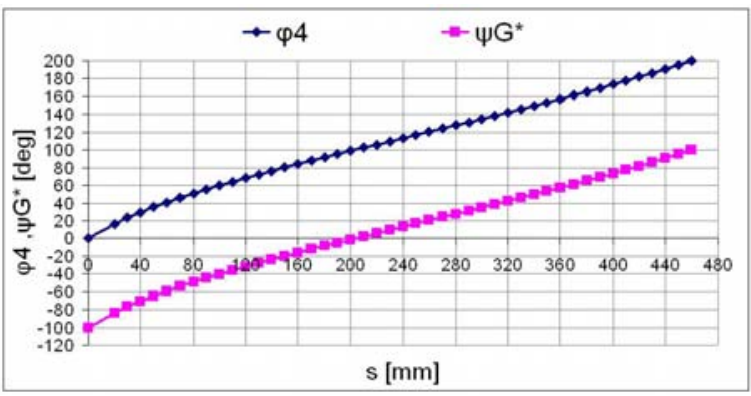

Fig. 9. The geared linkage displacement law $\varphi_{4}=\varphi_{4}(\mathrm{~s})$ and the gear linkage azimuth displacement law $\psi_{\mathrm{G}}{ }^{*}=\psi_{\mathrm{G}}{ }^{*}(\mathrm{~s})$.

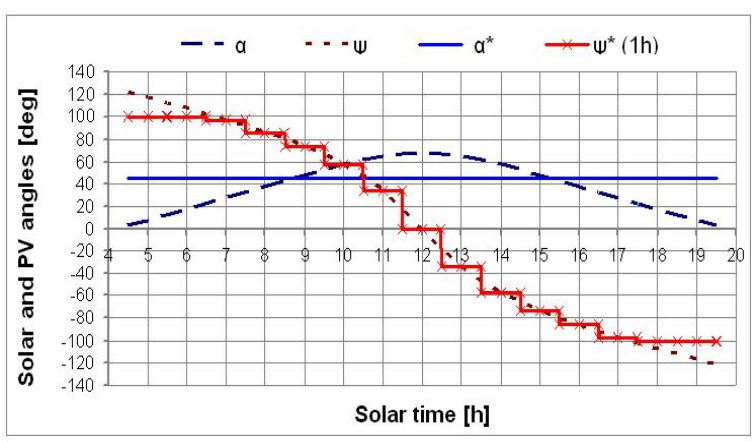

Fig. 10. Variations of sunray angles $\psi$ and $\alpha$ and variations of the PV corresponding angles $\psi^{*}$ and $\alpha^{*}$, during the Summer Solstice in Brasov/Romania.

The variations of the sunray angles: azimuth angle $\psi$ and the elevation angle $\alpha$ [5] are presented in Figure 10 and also the variations of PV's corresponding angles: azimuth 
$\psi^{*}$ (step-wise variation with $1 \mathrm{~h}$ duration/step) and elevation $\alpha^{*}=45^{\circ}$ during Summer Solstice $(\mathrm{N}=172)$, under clear sky conditions.

Using Figures 9 and 10, the function $\mathrm{s}=\mathrm{s}$ (solar time) can be estimated, as presented in Figure 11. Finally the variations of the input and output parameters of the tracked PV system are calculated and presented in Figure 12: $\mathrm{s}=\mathrm{s}$ (solar time) and $\psi^{*}=\psi^{*}($ solar time).

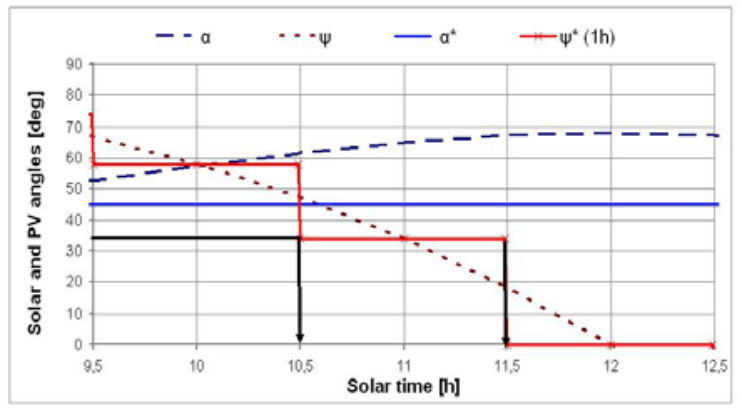

a)

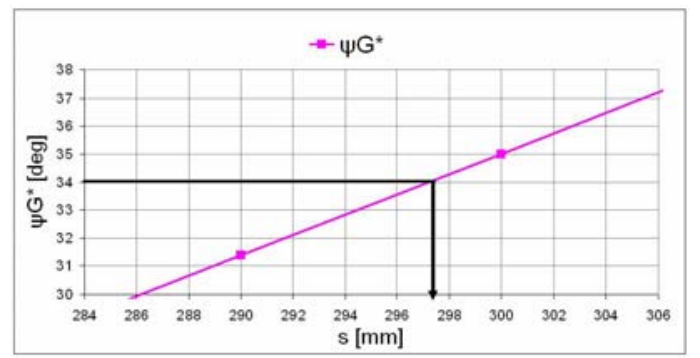

b)

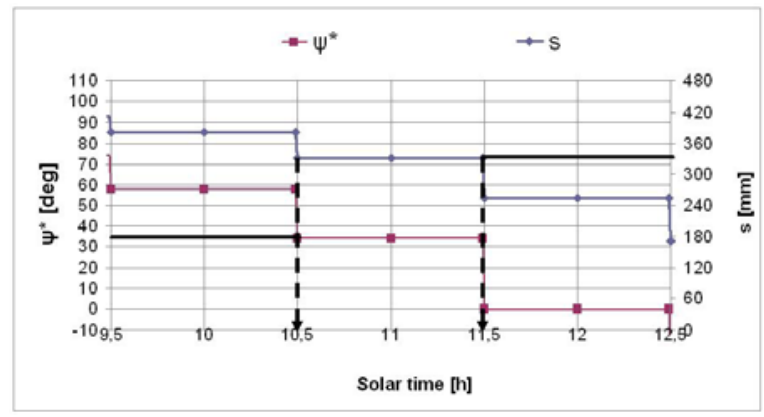

c)

Fig. 11. Details from: a) Fig. 10, b) Fig. 9 and c) for the evaluating the step variations: $\mathrm{s}=\mathrm{s}$ (solar time).

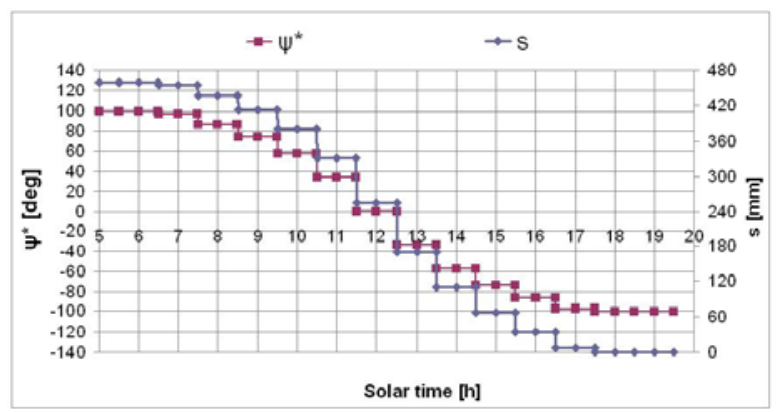

Fig. 12. The step variations of the actuator's linear displacement $\mathrm{s}=\mathrm{s}$ (solar time) and the angular displacement of the azimuth platform $\psi^{*}=\psi^{*}$ (solar time) during the Summer Solstice in Brasov/Romania.
The available direct solar radiation $\mathrm{B}$ and the direct solar radiation received on the PV platform $\mathrm{B}^{*}$ are calculated using the following equations [7]:

$$
\mathrm{B}=\mathrm{B}_{0} \exp \left[-\mathrm{T}_{\mathrm{R}} /(0.9+9.4 \sin \alpha)\right]
$$

where:

$$
B_{0}=1367 \cdot[1+0.0334 \cdot \cos (0.9856 \cdot N-2.27]
$$

$\mathrm{T}_{\mathrm{R}^{-}}$represents the turbidity factor for Brasov area, $\left(\mathrm{T}_{\mathrm{R}} \approx 3,[8]\right)$;

$\mathrm{N}-$ is the day number within a year.

The direct radiation that falls normally on the PV module surface can be estimated using the Lambert's law [5]:

$$
B_{P V}=B \cdot \cos v
$$

where: $v$ is the incidence angle [9]:

$$
\mathrm{v}=\cos ^{-1}\left(\cos \alpha \cdot \cos \alpha^{*} \cos \left(\psi-\psi^{*}\right)+\sin \alpha \cdot \sin \alpha^{*}\right)
$$

The variation of the available direct solar radiation and the radiation received on the PV module surface are presented in Figure 13. It can be observed that the radiation gained by the mono-axial tracker almost reaches the values of the available radiation; the difference is made by the constant elevation angle $\left(\alpha^{*}=45^{\circ}\right)$.

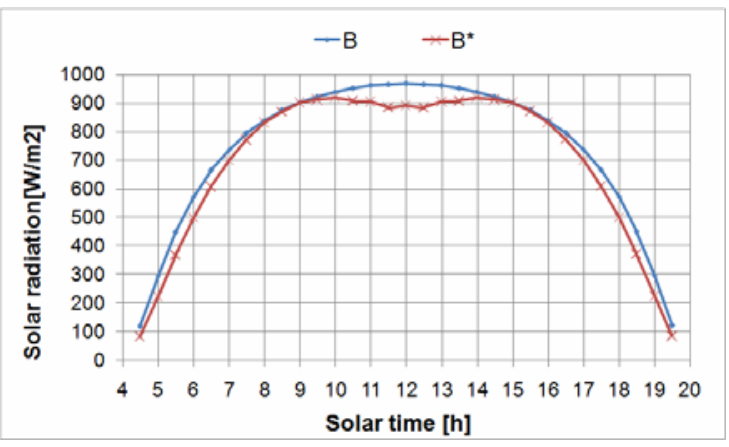

Fig. 13. Variations of the available direct solar radiation (B) and the direct radiation received by the PV platform $\left(\mathrm{B}^{*}\right)$, during the Summer Solstice in Brasov/Romania.

In order to evaluate the performance of the tracking system, the tracking efficiency is calculated [8]:

$$
\eta_{\text {tracking }}=\frac{E_{B^{*}}}{E_{B}}
$$

where: $E_{B}$ represents the energy of the available solar radiation $(\mathrm{B})$ :

$$
E_{B}=\int B d t
$$

and: $E_{B^{*}}$ represents the energy of the received direct solar radiation on the $\mathrm{PV}$ surface:

$$
E_{B^{*}}=\int B * d t
$$


The tracking efficiency of the mono-axial tracker, obtained during the Summer Solstice, is $94.42 \%$; i.e. the percentage from the available direct solar energy that falls on the PV platform.

\section{Conclusions}

The paper presents a new type of geared linkage used for building large azimuth strokes for photovoltaic modules. Depending on the internal speed ratio $\mathrm{i}_{0}$, the geared linkage can reach large angular strokes (over $250^{\circ}$ ); having good transmission angles and a reasonable cost.

The geared linkage's geometric model is defined by: the angular stroke $\left(\Delta \varphi_{4}\right)$, the rocker length $(r)$, the base length (b), the linear actuator stroke $(\Delta s)$ and is the angle between the initial start position of gear 4 and the South direction $\left(\psi_{0}\right)$. Using this model a kinematical synthesis algorithm is presented and simulation are made for a numerical application (where $\Delta \varphi_{4}=200^{\circ}$ and the minimum allowed transmission angle $\gamma_{\mathrm{m}} \geq 30^{\circ}$ are imposed)

The kinematical synthesis leads to the optimal geometrical dimensions of the geared linkage. Based on these dimensions, the mechanism's and the azimuth geared linkage's transmission laws are found: $\varphi_{4}=\varphi_{4}(\mathrm{~s})$ and respectively $\psi_{\mathrm{G}}{ }^{*}=\psi_{\mathrm{G}} *(\mathrm{~s})$; based on the last law and on the step azimuth PV program, $\psi^{*}=\psi^{*}$ (solar time), the tracking law for the PV platform, $\mathrm{s}=\mathrm{s}$ (solar time), was estimated, during the summer solstice day, in the Brasov/Romania area.

For a mono-axial tracking system, using the proposed mechanism and elevation $\alpha^{*}=45^{\circ}$, the tracking efficiency was estimated at a value of $94.42 \%$ for the Summer Solstice.

\section{Acknowledgement}

This paper is supported by the Sectoral Operational Programme Human Resources Development (SOP HRD), financed from the European Social Fund and by the Romanian Government under the contract number POSDRU/88/1.5/S/5932.

\section{References}

[1] D. J Goswami. et al., Principles of Solar Engineering, CRC, 2000.

[2] Messenger R., Ventre J., Photovoltaic System Engineering, CRC Press, London, 2000

[3] S. Blackman, R. Popoli, Design and Analysis of Modern Tracking Systems, Artech House Publishers, Boston, 2000.

[4] I. Vişa, et al: The Synthesis of a Linkage with Linear Actuator for Solar Tracking with Large Angular Stroke, in: Proc. of EUCOMES, Cassino, Italy, 2008, p. 457-464.

[5] Vatasescu M., et al: New Solar Angles and their Corresponding Tracking Systems Efficiency, Renewable Energy and Power Quality Journal, No.8, April, 2010

[6] Burduhos, B. et al.: The Synthesis of a Planar Linkage Used to Drive the Daily Motion of a Solar Tracker, MTM 2008 International Conference MTM, p. 17 - 20, 2008.

[7] M. Meliss, Regenerative Energie-quellen Praktikum, Springer, Berlin (1997).

[8] M. M. Vătăşescu, et al: Atmospheric Pollution Evaluation in Brasov Romania Based on Turbidity Factor Analysis, ICAMBES 2010, Transilvania University Press, p. 106

[9] Visa, I., et al: On the Incidence Angle Optimization of the Dual-Axis Solar Tracker. 11th Inter. Research/Expert Conf. TMT, Hamammet, Tunisia, p. 1111-1114, 2007. 